This item was submitted to Loughborough's Research Repository by the author.

Items in Figshare are protected by copyright, with all rights reserved, unless otherwise indicated.

\title{
New customized elbow orthosis made by additive manufacturing
}

PLEASE CITE THE PUBLISHED VERSION

https://www.springer.com/gp/book/9783030123451

PUBLISHER

Springer

VERSION

AM (Accepted Manuscript)

PUBLISHER STATEMENT

The final authenticated version is available online at https://doi.org/10.1007/978-3-030-12346-8_46.

LICENCE

CC BY-NC-ND 4.0

\section{REPOSITORY RECORD}

Campbell, lan, T. Ingrassia, V. Nigrelli, and V. Ricotta. 2019. "New Customized Elbow Orthosis Made by Additive Manufacturing". Loughborough University. https://hdl.handle.net/2134/33820. 


\title{
New customized elbow orthosis made by Additive Manufacturing
}

\author{
Campbell R.I. ${ }^{1}$ Ingrassia T. ${ }^{2, *}$, Nigrelli V. ${ }^{2}$, Ricotta V. ${ }^{2}$ \\ ${ }^{1}$ Loughborough Design School, Loughborough University, Loughborough LE11 3TU, UK \\ ${ }^{2}$ Università degli Studi di Palermo, Dipartimento dell'Innovazione Industriale e Digitale - Ingegneria \\ Chimica, Gestionale, Informatica, Meccanica, Viale delle Scienze - 90128 Palermo, Italy \\ * Corresponding author: Tel.:+39 091 23897263; E-mail address: tommaso.ingrassia@unipa.it
}

\begin{abstract}
Orthoses are additional devices that help people with disabilities.

The focus of this work is the design and manufacture of a new customized elbow orthosis completely made by Additive Manufacturing (AM). One of the innovative characteristic of the device is the use of torsion springs that simulate the action of physiotherapists during exercises for patient rehabilitation. Parametric modeling approach based on generative algorithms was used to design the device.

Finally, FEM analyses have been performed to validate the design.
\end{abstract}

Keywords: Additive Manufacturing; 3D acquisition; Computer Aided Engineering.

\section{Introduction}

Orthoses are external medical devices used in orthopedics in the treatment of some pathologies to correct a functional anomaly, but which don't replace missing anatomic parts. These devices limit the relative movements in a joint affected, for example, by trauma, arthrosis, ligament sprains or that has been subjected to surgery. For patients with disabilities that induce functional limitations, orthoses are employed to apply forces on the body for biomechanical needs by helping patients during walking and limb movement.

Orthoses can be prefabricated or customized. The prefabricated ones are less expensive and readily available on the market. Customized orthoses, instead, are better adapted to the patient's body than the prefabricated devices and ensure better performances [1,2].

The fundamental aspects in the design of customized orthoses are functionality, ergonomics and aesthetics. Designing an orthosis by combining all three of these aspects means being able to make a prosthetic device that fully meets the needs of patients. Additive Manufacturing (AM) is a suitable technology to make customized orthoses, especially for its ability to realize complex shapes in a short time and with 
lower costs than manual fabrication [3-7]. Most of the orthoses on the market are prefabricated devices that can be adapted to different sizes by means of appropriate bands or other methods. Most of the commercial orthoses are only partially realized with $\mathrm{AM}$ technologies and then assembled to constitute the final device, with a consequent increase in production phases and costs depending on the particular orthosis to be made. In the literature there are different works about wrist orthoses [8], foot orthoses [9] and hip orthoses [10], but there is not much information about the customized elbow orthosis completely manufactured using techniques of AM.

For this reason, this work focuses on the study of an elbow orthosis.

The aim of this work is the design of a new customized elbow orthosis completely manufacturable by $\mathrm{AM}$ and the implementation of generative algorithms for parametric modeling and creation of 3D patterns to be adapted to the CAD model.

The present work has been divided into four main steps. The first step consists of the $3 \mathrm{D}$ acquisition process and then the realization of the CAD model of the arm of a patient. In the second step, the CAD modeling of the customized orthosis has been performed. During this step, generative algorithms have been developed for the realization of a Voronoi tessellation and for the creation of patterns with different modules in order to better customize the device. In the third step, the prototype of the orthosis has been realized using AM techniques. In particular, the Selective Laser Sintering (SLS) process with PA 2200 material has been used. Finally, results obtained by the FEM analyses performed during the last step were used to validate the design.

\section{New model of elbow orthosis}

The basic idea of this work is to design of a new customized elbow orthosis manufactured entirely by AM technology, able to help patients during the rehabilitation phase. One of the fundamental exercises during the rehabilitation of the patient is the execution of flexion and extension movements of the forearm (Figure 1) that help to slowly return to the full functionality and mobility of the arm. Starting from the neutral position (angle between arm and forearm of $90^{\circ}$ ) during the flexion and extension movements the physiotherapist opposes resistance. After, to return to the neutral position, the patient is eased by the physiotherapist.

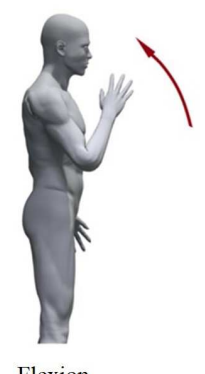

Flexion

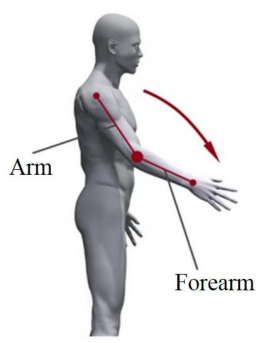

Extension

Fig. 1 - Flexion and extension movements of the forearm 
In order to simulate this rehabilitation exercise, with no support of physiotherapists, it was decided to apply two torsion springs to the orthosis that oppose resistance during the flexion and extension movements and help the patient to move the arm until to reach the neutral position. In this way the patient can carry out itself the rehabilitation exercise.

The spring have been chosen taking into account that the patient, during the rehabilitation phase, should not lift weights more than $5 \mathrm{~kg}$.

\section{3D acquisition and CAD modeling of the arm}

In order to model correctly the shape of the patient's arm, and in particular the elbow area, it was decided to reproduce a copy of it using a plaster cast.

A direct acquisition of the patient's arm, in fact, could lead to a poor model or require very complex post-process phases due to the impossibility of keeping the patient's arm perfectly fixed during the whole scanning phase.

To avoid these drawbacks, a plaster cast of the patient's arm has been made. Once the cast has been made, it was acquired using a 3D scanner, the ZScanner 800 by ZCorporation.

This scanner has been chosen for its main features: the self-orienting and the possibility of moving the object during the scan. Thanks to the self-orienting feature, the scan system does not need a fixed position tripods, voluminous mechanical arms or external positioning devices, which make the acquisition of some hidden surfaces difficult or almost impossible.

These characteristics have facilitated greatly the acquisition and post-processing phase, reducing the time required to obtain the digital model of the arm.

The model obtained from the scan is only the part related to the elbow area. In order to model the remaining part of arm, a series of measurements of some sections of the arm have been made at different positions. Subsequently, it has been possible reconstructed the entire model of the arm by means of a loft function.

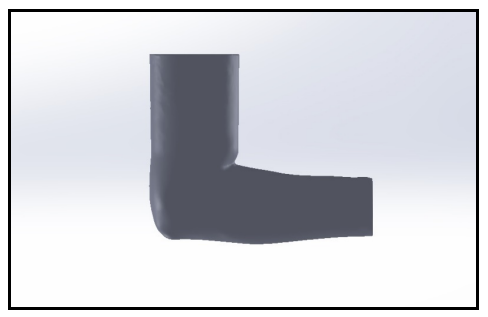

Fig. 2 - CAD Model of the arm surface 


\section{CAD modeling of the orthosis}

Customized orthoses, besides to adapt better to the patient, also allow optimizing their stiffness and flexibility to inhibit some movements and to integrate new morphological characteristics.

The modeling of a customized orthosis starts from the CAD model of the patient's arm. To ensure a good level of ergonomics, the orthosis should be perfectly adapted to the arm. However, it is also necessary to consider the presence of swelling of the patient's elbow and ensuring that the orthosis does not compress excessively, in order to avoid tissue inflammation or other undesired effects.

Regarding the functionality, one of the aspects to be taken into consideration is the possibility for the patient to wear and remove the orthosis easily and independently, with no discomfort at the elbow.

In order to meet the aforementioned ergonomics and wearability requirements, starting from the reconstructed surface of the arm, a $2 \mathrm{~mm}$ offset has been applied, ensuring a small clearance between the arm and the orthosis; moreover, two openings have been made, so to allow both to wear the orthosis without problems and to increase the adaptability.

To ensure a suitable stiffness of the orthosis, but at the same time don't add weigh to the entire device, preliminary, a thickness of $4 \mathrm{~mm}$ of the structure has been chosen. This value of thickness is usually adopted in commercial orthoses.

The next step was the conversion of the model into NURBS surfaces and then into a CAD model.

The relative rotation between the arm and forearm during the extension and flexion movements is ensured by a hinged mechanism.

Obtained the basic CAD model of the orthosis, all the components have been modelled and assembled into the final CAD model of the orthosis by SolidWorks software. (Figure 3).

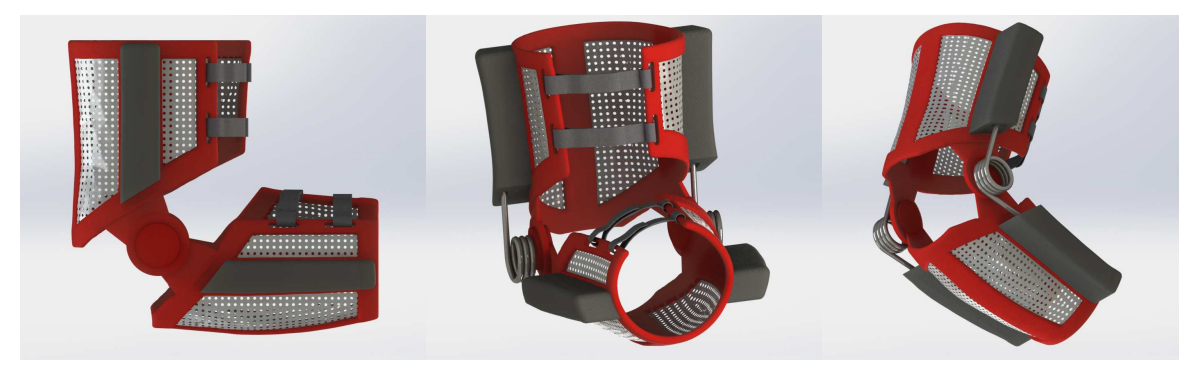

Fig. 3 - CAD model of the elbow orthosis 


\subsection{Generative algorithms}

Generative algorithms allow finding solutions to problems that can be encountered with the classic CAD systems when complex shapes, especially when these would be realized using AM techniques [12-16], must be modelled.

In this work, generative algorithms have been implemented for the modeling of nonstructural parts of the orthosis that, in addition to lightening the structure, must make the orthosis pleasant from an aesthetic point of view.

Two different algorithms have been developed with Grasshopper [17,18]; the first allows the creation of patterns on complex surfaces, the latter allows to realizing a Voronoi tessellation.

\subsubsection{Generative algorithm for creating patterns}

The developed algorithm allows users to create patterns on complex surfaces, so customizing the orthosis with different decorative geometries.

The basic function generates different parallelepipeds on the surface, used as target objects, and maps on these the geometry to repeat.

The target parallelepipeds have been generated to morph the component using the "Surface Box" function [17], adapting them to a surface based on the interval indicated by the surface domain and the height of the parallelepiped.

The final result is the pattern created on the surface that is perfectly adapted to it and that follows its morphology on a regular basis.

Figure 4 shows the block diagram of the developed algorithm.

It is possible to modify the number of elements along the parameterization directions $(\mathrm{U}$ and $\mathrm{V}$ ) and also to change the basic geometry.

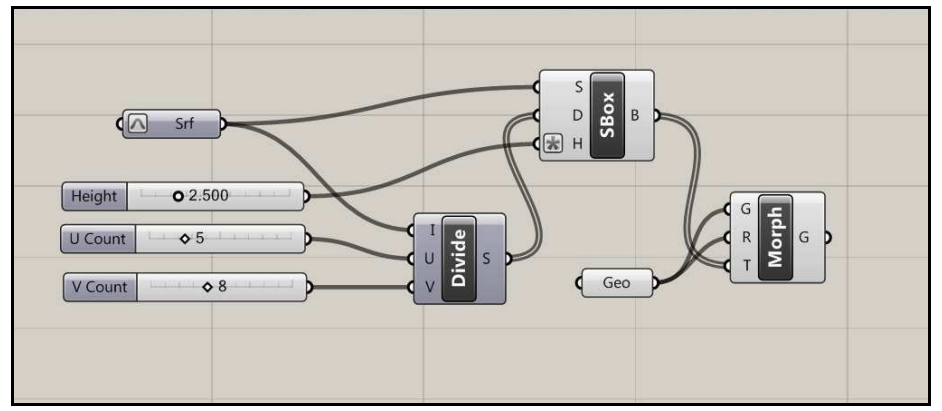

Fig. 4 - Block diagram of the implemented generative algorithm

In figure 5 basic component to repeat (left) and pattern created with generative algorithm are shown. 
In figure 6 some examples of patterns created with this algorithm and applied to the elbow orthosis are shown.

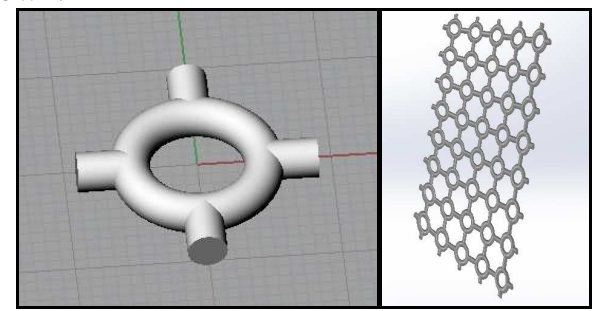

Fig. 5 - Example of basic component to repeat (left) and pattern created with generative algorithm
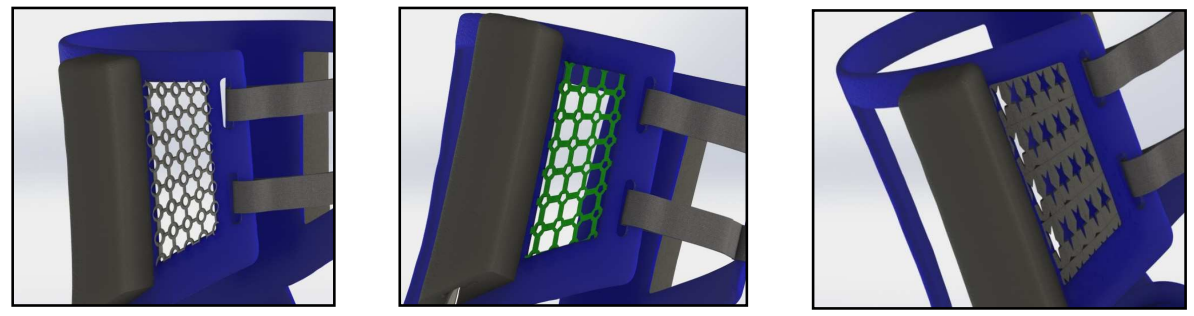

Fig. 6 - Examples of patterns that can be created and applied to the orthosis

\subsubsection{Voronoi tessellation by generative algorithm}

Because not all the commercial software allow managing independently the input parameters for the creation of Voronoi tessellations, a generative algorithm has been developed to manage these parameters (such as number of points, width of each polygon of the tessellation, etc..) and to model, parametrically, a Voronoi structure in the custom orthosis.

The Voronoi tessellation is initially created on a surface in a plane and is subsequently mapped onto the surface of the orthosis. After, with specific functions, the mesh is generated and exported.

The block diagram of the generative algorithm developed in Grasshopper for the realization of the Voronoi tessellation to be applied for the customization of the elbow orthosis is shown in figure 7.

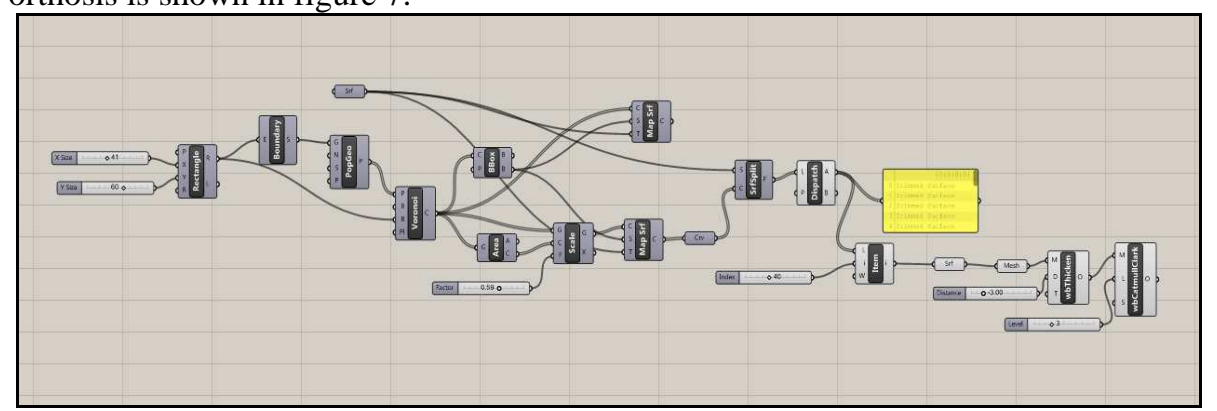

Fig. 7 - Block diagram of the implemented generative algorithm for Voronoi tessellation 
The creation of the Voronoi tessellation starts from a set of points that represent the centre of the polygons of the tessellation; after, through the Voronoi function, a twodimensional Voronoi tessellation has been created according to the set of points.

This structure is mapped onto the surface of the orthosis through the "Map to Surface" function [17], adapting itself to the morphology of the surface.

The last step of the algorithm is the creation of mesh and the smoothing of the structure so to eliminate the sharp edges.

In figure 8 the complete CAD model of the orthosis with the Voronoi tessellation is shown.

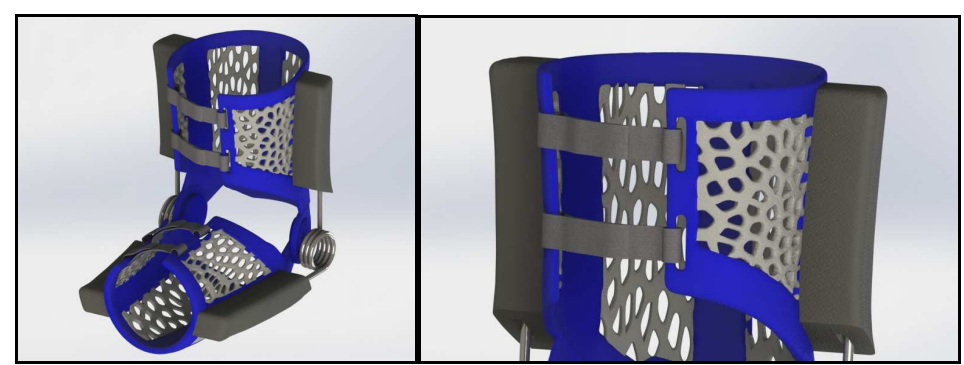

Fig. 8 - CAD model of the elbow orthosis with Voronoi tessellation

\section{Prototype realization by Additive Manufacturing}

The designed elbow orthosis has been produced in PA 2200 by a SLS (Selective Laser Sintering) 3D printer. As case study, only the model of the orthosis with the Voronoi tessellation has been realized.

PA 2200 is a fine white powder based on polyamide, used for the manufacture of fully functional pieces with high surface quality and exposed to high thermal or mechanical stresses [11].

The EOS FORMIGA P100 printer (Figure 9) has been used, which allows the production of small series and customized products with fully functional complex geometry.

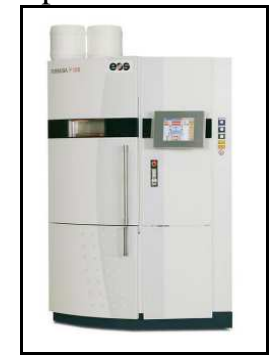

Fig. 9 - FORMIGA P100 3D printer 
Figures 10-11 show some images of the prototype from which is possible to see the good surface finish obtained with this AM technology.

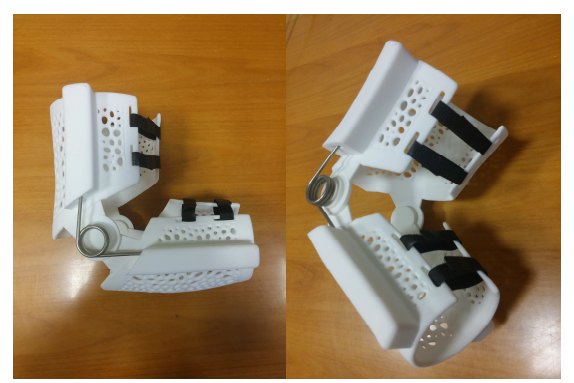

Fig. 10 - Final prototype

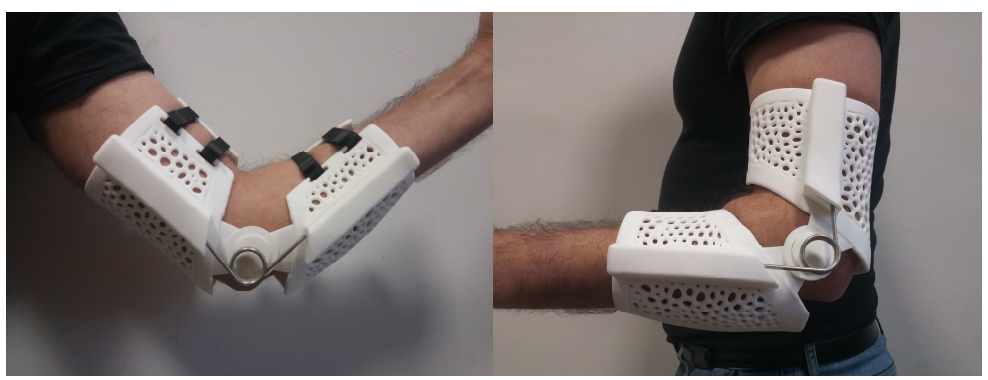

Fig. 11 - Final prototype put on by patient

\section{FEM analysis}

FEM analyses have been performed to verify the structural strength of the prototype [19].

Only the structural parts of the orthosis have been analysed.

The arm has been considered as a rigid body. The area near the elbow has been not considered to simplify the FEM model, always ensuring the reliability of the results. The simulations have been performed to verify the stress state of the orthosis during flexion and extension, applying a test load of $150 \mathrm{~N}$.

Although the maximum load in normal conditions of use of an elbow orthosis should be less than $50 \mathrm{~N}$, a much higher test load $(150 \mathrm{~N})$ was chosen to test the model in very hard working conditions.

The model has been meshed with about 2.000.000 Solid 164 elements [20]. Successively the boundary conditions have been set. In particular, a frictional contact has been imposed at the orthosis and arm interface, a frictionless contact, instead, has 
been imposed at the torsion springs and the supports interfaces. Finally, a fixed constraint has been applied to the faces of the arm (in the upper part) which prevents them from moving or deforming. The strips have been modelled as spring elements.

Table 1. Mechanical properties of PA 2200.

\begin{tabular}{|c|c|c|c|}
\hline & Tensile strength (MPa) & Elongation at break (\%) & Tensile modulus (MPa) \\
\hline PA 2200 & 48 & 24 & 1700 \\
\hline
\end{tabular}

\section{$\underline{\text { Extension }}$}

Figure 12 shows Von Mises stress maps.
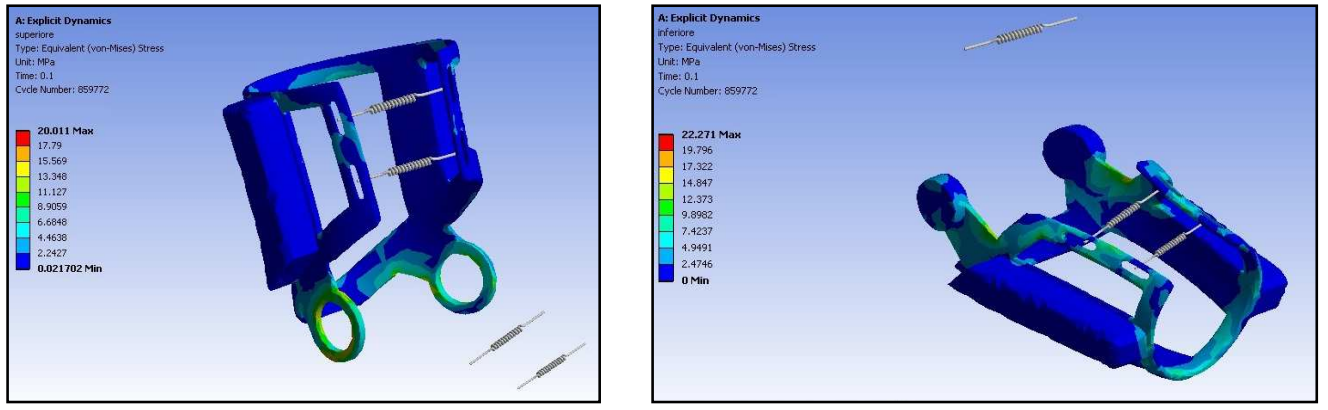

Fig. 12 - Maps: Von Mises stress

It can be observed that all the maximum values of the deformation and strains are lower than the corresponding limit values. In particular, the maximum value of the Von Mises stress is $22.27 \mathrm{MPa}$, less than half of the tensile strength of the material (48 MPa).

\section{Flexion}

$\overline{\text { Figure } 13}$ shows Von Mises stress maps.
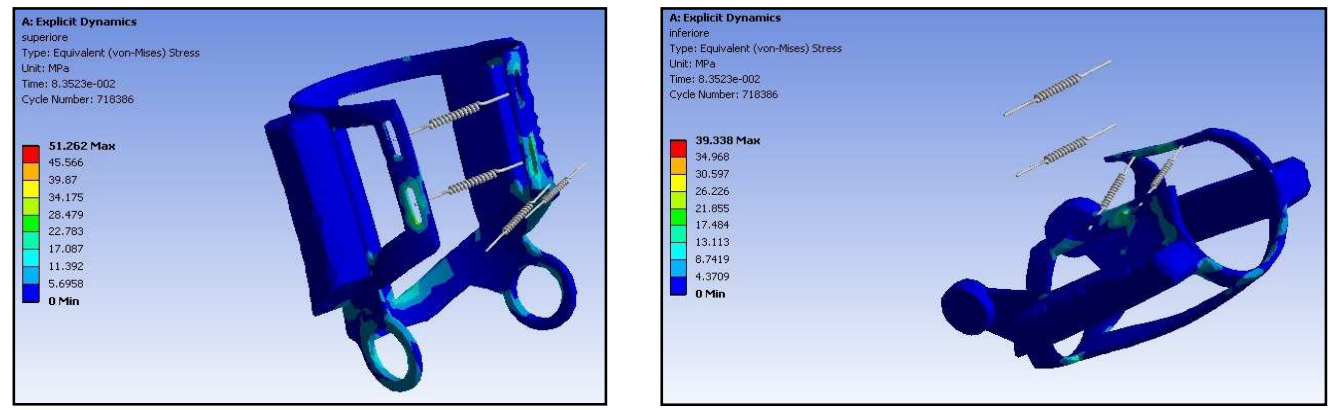

Fig. 13 - Maps: Von Mises stress 
Also in this case, it can be observed that all the maximum values of the deformation and strains are lower than the corresponding limit values. In particular, a maximum elongation value of $2.1 \%$ and a maximum value of the Von Mises equivalent stress of $41 \mathrm{MPa}$ have been obtained.

\section{Conclusions}

In the work a method has been perfected for the design of a custom elbow orthosis entirely manufacturable by additive techniques. To this aim, generative algorithms for parametric modeling and creation of 3D patterns have been also developed that can solve some of typical problems of modeling for Additive Manufacturing.

The first step of the work was related to the modeling, by means of reverse engineering techniques, of the arm of a patient and the subsequent CAD modeling of the structure of the custom elbow orthosis.

Subsequently, to improve the aesthetic appearance of the orthosis, two algorithms have been implemented for the creation of $3 \mathrm{D}$ patterns and Voronoi tessellations for not structural parts.

Generative algorithms developed have been tested and proved to be robust, efficient and able to overcome some drawbacks of commercial software.

Subsequently, a prototype of the elbow orthosis with Voronoi tessellation has been realized by means of the SLS technology.

In the last part FEM analyses have been carried out considering the movements of flexion and extension of the arm and obtained results were used to validate the design.

\section{References}

1. Mohd. Javaid, Abid Haleem, Additive manufacturing applications in medical cases: A review literature based, Alexandria Journal of Medicin (2017).

2. Sunpreet Singh, Seeram Ramakrishna, Biomedical applications of additive manufacturing: Present and future, Current Opinion in Biomedical Engineering 2017, 2:105115 .

3. Roland K. Chen, Yu-an Jin, Jeffrey Wensman, Albert Shih, Additive manufacturing of custom orthoses and prostheses-A review, Additive Manufacturing 12 (2016) 77-89

4. Wei Gao, Yunbo Zhang, Devarajan Ramanujan, Karthik Ramania, Yong Chen, Christopher B. Williams, Charlie C.L. Wang, Yung C. Shin, Song Zhang, Pablo D. Zavattieri, The status, challenges, and future of additive manufacturing in engineering, Computer-Aided Design 69 (2015) 65-89.

5. Samuel H. Huang, Peng Liu, Abhiram Mokasdar, Liang Hou, Additive manufacturing and its societal impact: a literature review, Int J Adv Manuf Technol (2013) 67:1191-1203 DOI 10.1007/s00170-012-4558-5.

6. H. Bikas, P. Stavropoulos, G. Chryssolouris, Additive manufacturing methods and modelling approaches: a critical review, Int J Adv Manuf Technol DOI $10.1007 / \mathrm{s} 00170-015-7576-2$ 
7. Cauê G. Mançanares, Eduardo de S. Zancul, Juliana Cavalcante da Silva, Paulo A. Cauchick Miguel, Additive manufacturing process selection based on parts' selection criteria, Int J Adv Manuf Technol (2015) 80:1007-1014 DOI 10.1007/s00170-0157092-4.

8. Abby M Paterson, Ella Donnison, Richard J Bibb, R Ian Campbell, Computer-aided design to support fabrication of wrist splints using 3D printing: A feasibility study, December 2014 Hand Therapy 19(4):102-113 DOI 10.1177/1758998314544802.

9. Fantini M, De Crescenzio F, Brognara L, Baldini N, Design and Rapid Manufacturing of a customized foot orthosis: a first methodological study, Springer International Publishing AG (2017) Advances on Mechanics, Design Engineering and Manufacturing, Lecture Notes in Mechanical Engineering, DOI 10.1007/978-3-31945781-9_46.

10. Rodrigo Munhoz, Cícero André da Costa Moraes, Harki Tanaka, Maria Elizete Kunkel, A digital approach for design and fabrication by rapid prototyping of orthosis for developmental dysplasia of the hip, Research on Biomedical Engineering, (2016) Volume 32, Number 1, p. 63-73, DOI 10.1590/2446-4740.00316.

11. Sunpreet Singh, Seeram Ramakrishna, Rupinder Singh, Material issues in additive manufacturing: A review, Journal of Manufacturing Processes 25 (2017) 185-200.

12. Daniel R. Eyers, Andrew T. Potter, Industrial Additive Manufacturing: A manufacturing systems perspective, Computers in Industry 92 (2017) 208-218.

13. Christoph Klahn, Bastian Leutenecker, Mirko Meboldt, Design Strategies for the Process of Additive Manufacturing, Procedia CIRP 36 (2015) 230 - 235.

14. Sebastian Hällgren, Lars Pejryd, Jens Ekengren, (Re)Design for Additive Manufacturing, Procedia CIRP 50 ( 2016 ) $246-251$

15. M.Srinivas, B.Sridhar Babu, A Critical Review on Recent Research Methodologies in Additive Manufacturing, Materials Today: Proceedings 4 (2017) 9049-9059

16. Mary Kathryn Thompson, Giovanni Moroni, Tom Vaneker, Georges Fadel, R. Ian Campbell, Ian Gibson, Alain Bernard, Joachim Schulz, Patricia Graf, Bhrigu Ahuja, Filomeno Martina, Design for Additive Manufacturing: Trends, opportunities, considerations, and constraints, CIRP Annals - Manufacturing Technology (2016) .

17. ZUBIN KHABAZI, ALGORITMI GENERATIVI con GRASSHOPPER.

18. Sivam Krish, A practical generative design method.

19. Cappello, F., Ingrassia, T., Mancuso, A., Nigrelli, V., Methodical redesign of a semitrailer, (2005) WIT Transactions on the Built Environment, 80, pp. 359-369.

20. ANSYS Structural Analysis Guide. 